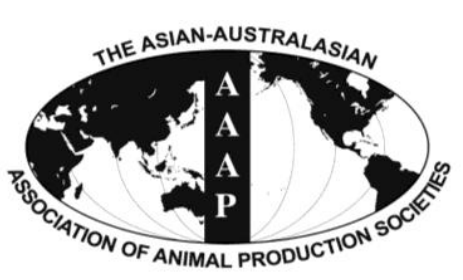

Open Access

Asian Australas. J. Anim. Sci.

Vol. 27, No. 1 : 83-92 January 2014

http://dx.doi.org/10.5713/ajas.2013.13369

www.ajas.info

pISSN $1011-2367$ elSSN 1976-5517

\title{
Effects of Dietary Protein Levels for Gestating Gilts on Reproductive Performance, Blood Metabolites and Milk Composition
}

\author{
Y. D. Jang ${ }^{1}$, S. K. Jang, D. H. Kim, H. K. Oh ${ }^{2}$, and Y. Y. Kim* \\ School of Agricultural Biotechnology, Seoul National University, 1 Gwanak-ro, Gwanak-gu, Seoul 151-921, Korea
}

\begin{abstract}
This experiment was conducted to evaluate the effects of dietary CP levels in gestation under equal lysine content on reproductive performance, blood metabolites and milk composition of gilts. A total of 25 gilts ( $\mathrm{F}_{1}$, Yorkshire $\times$ Landrace) were allotted to 4 dietary treatments at breeding in a completely randomized design, and fed 1 of 4 experimental diets containing different CP levels $(11 \%, 13 \%, 15 \%$, or $17 \%)$ at $2.0 \mathrm{~kg} / \mathrm{d}$ throughout the gestation. Body weight of gilts at $24 \mathrm{~h}$ postpartum tended to increase linearly $(\mathrm{p}=$ 0.09 ) as dietary CP level increased. In lactation, backfat thickness, ADFI, litter size and weaning to estrus interval (WEI) did not differ among dietary treatments. There were linear increases in litter and piglet weight at $21 \mathrm{~d}$ of lactation $(\mathrm{p}<0.05)$ and weight gain of litter $(\mathrm{p}<0.01)$ and piglet $(\mathrm{p}<0.05)$ throughout the lactation as dietary CP level increased. Plasma urea nitrogen levels of gilts in gestation and at $24 \mathrm{~h}$ postpartum were linearly elevated as dietary CP level increased ( $\mathrm{p}<0.05$ ). Free fatty acid (FFA) levels in plasma of gestating gilts increased as dietary CP level increased up to $15 \%$, and then decreased with quadratic effects $(15 \mathrm{~d}, \mathrm{p}<0.01 ; 90 \mathrm{~d}, \mathrm{p}<0.05)$, and a quadratic trend $(70 \mathrm{~d}, \mathrm{p}=0.06)$. There were no differences in plasma FFA, glucose levels and milk composition in lactation. These results indicate that increasing dietary $\mathrm{CP}$ level under equal lysine content in gestation increases $\mathrm{BW}$ of gilts and litter performance but does not affect litter size and milk composition. Feeding over 13\% CP diet for gestating gilts could be recommended to improve litter growth. (Key Words: Gilt, Protein, Reproductive Performance, Milk Composition, Litter Growth)
\end{abstract}

\section{INTRODUCTION}

Protein and amino acids play crucial roles in reproductive performance of sows (Kim et al., 2009). Pettigrew and Yang (1997) reported that an adequate supply of protein and amino acids during gestation allowed sows to maintain their productivity, and that high body protein content of sows could maximize milk production and subsequent reproductive performance. NRC (1998) recommended $12.9 \% \mathrm{CP}$ in gestation diets for gilts (average $125 \mathrm{~kg}$ of $\mathrm{BW}$ at breeding). However, modern gilts, genetically improved to have large litter and high milk production, require more nutrients to carry on normal

\footnotetext{
* Corresponding Author: Y. Y. Kim. Tel: +82-2-880-4801, Fax: +82-2-878-5839, E-mail: yooykim@snu.ac.kr

${ }^{1}$ Animal and Food Sciences, University of Kentucky, Lexington, USA.

2 Department of Food and Nutrition, Jangan University, Hwaseong, Gyeonggi-do, Korea.

Submitted Jun. 28, 2013; Accepted Aug. 13, 2013; Revised Sept. 12, 2013
}

reproductive cycle and body maturation (Boyd et al., 2000).

In previous studies, restricted dietary protein for gestating gilts did not affect litter size and litter birth weight but caused detrimental effects on litter weight gain although sufficient protein was provided during lactation (Baker et al., 1970; Kusina et al., 1999b). On the other hand, high dietary protein for gestating gilts increased litter weight and weight gain (Mahan, 1998), and elevated milk yield as well (Kusina et al., 1999b).

Recently, Zhang et al. (2011) reported sows fed gestation diets containing higher lysine levels (0.65 to 0.75\%) than NRC (1998) recommendation under the same content of CP showed improvements in reproductive performance and litter growth, indicating lysine content in the gestation diets need to be increased. However, there is no evidence for the effect of dietary CP levels under high lysine content in gestation for gilts. Even though lysine has been considered the first-limiting amino acid in cornsoybean meal diets for sows in gestation and lactation, the other essential amino acids play important roles in the 
efficiency of protein utilization by gestating sows (Kim et al., 2009). Increased supply of dietary CP in gestation could allow gilts to consume adequate amino acids to maximize reproductive performance and ensure body development. Therefore, the objective of this experiment was to evaluate the effect of dietary CP levels for gestating gilts on reproductive performance including milk composition and their progeny's appearance.

\section{MATERIALS AND METHODS}

\section{Animal management, housing and experimental diets}

At $180 \mathrm{~d}$ of age and approximately $105 \mathrm{~kg} \mathrm{BW}$, a total of 30 gilts $\left(\mathrm{F}_{1}\right.$, Yorkshire $\times$ Landrace, Darby, Icheon, Korea) were housed in environmentally controlled pens $(2.5 \times 3.5$ $\mathrm{m}^{2}$ ) with 3 gilts per pen. The gilts were given ad libitum access to feed and water through a feeder and a water cup nipple. At $220 \mathrm{~d}$ of age, the gilts were moved into individual gestation stalls $\left(2.40 \times 0.64 \mathrm{~m}^{2}\right)$ and fed a total of $2 \mathrm{~kg} / \mathrm{d}$ of a commercial gestation diet twice daily. After estrus check at approximately $240 \mathrm{~d}$ of age and $140 \mathrm{~kg}$ of $\mathrm{BW}$, the gilts were artificially inseminated (Darby AI center, Choongju, Korea) by 3 times at $12 \mathrm{~h}$ interval. Then, 25 gilts were selected and allotted to 1 of 4 dietary treatments in a completely randomized design. Four experimental diets containing different CP levels of $11 \%, 13 \%, 15 \%$, and $17 \%$ were provided to the gilts during gestation. Each diet in gestation contained $3,265 \mathrm{kcal}$ of $\mathrm{ME} / \mathrm{kg}, 0.74 \%$ of total lysine and the assigned content of $\mathrm{CP}$, respectively. The protein content was adjusted with corn, soybean meal and corn gluten meal to meet each assigned $\mathrm{CP}$ level of treatments. A lactation diet containing 3,269 kcal of ME/kg, and $1.08 \%$ of total lysine was provided after farrowing regardless of gestation treatments. All nutrients met or exceeded NRC (1998) nutrient requirement estimates. The formula and chemical composition of experimental diets are presented in Table 1.

Gestating gilts were housed in individual stalls $\left(2.40 \times 0.64 \mathrm{~m}^{2}\right)$ which were installed on a concrete floor in a temperature-controlled room with automatic fans and individually fed a total of $2.0 \mathrm{~kg} / \mathrm{d}$ divided equally between two meals fed at 08:00 and 16:00 during the entire gestation period. Pregnancy was confirmed on $28 \mathrm{~d}$ of gestation via ultrasound (Eagle scan, Dong Jin BLS Co, LTD., Gwangju, Korea). After $110 \mathrm{~d}$ of gestation, all gilts were moved into farrowing crates $\left(2.5 \times 1.8 \mathrm{~m}^{2}\right)$ and housed until weaning.

After parturition, the lactation diet was provided and gradually increased from $1.0 \mathrm{~kg} / \mathrm{d}$ by $0.5 \mathrm{~kg} / \mathrm{d}$ until $5 \mathrm{~d}$ postpartum with a free access to water. From $5 \mathrm{~d}$ postpartum, feed and water were provided ad libitum to primiparous sows. Within $24 \mathrm{~h}$ postpartum, Fe-dextran (150 ppm) injection, ear notching, needle teeth clipping and tail docking were practiced to each piglet. Piglets were cross- fostered across treatments within $3 \mathrm{~d}$ after birth to balance suckling intensity across sows with equalization of litter size, and thus to minimize any impact of initial litter size potentially affecting litter growth.

\section{Data collection}

Body weight and backfat thickness of gilts were measured at mating, $110 \mathrm{~d}$ of gestation, $24 \mathrm{~h}$ postpartum and $21 \mathrm{~d}$ of lactation. An ultrasound device (Lean-meter, Renco Corp., Minneapolis, US) was used for measuring backfat thickness at $\mathrm{P}_{2}$ position (mean value from both sides of the last rib and $65 \mathrm{~mm}$ away from the backbone). The numbers of total born, born alive, stillborn and mummified piglets were recorded within $24 \mathrm{~h}$ postpartum and the number of pigs was recorded after cross-fostering and at 21 $\mathrm{d}$ of lactation. Lactation feed intake was recorded weekly. Piglet weight was recorded at $24 \mathrm{~h}$ postpartum, after crossfostering and at $21 \mathrm{~d}$ of lactation. Litter weight was calculated by summing the individual piglet weights. Weaning to estrus interval (WEI) was determined by monitoring for estrus from 3 to $10 \mathrm{~d}$ after weaning.

\section{Sample collections and analysis}

For the initial, blood samples were randomly taken from 11 gilts immediately before mating. Colostrum, milk and blood samples were collected from 5 sows with 10 to 12 piglets from each treatment and 5 piglets (1/sow) were used to collect blood. Blood samples were collected from gilts through jugular vein into EDTA tubes at 15, 35, 70, 90, and $110 \mathrm{~d}$ of gestation as well as $24 \mathrm{~h}$ and $21 \mathrm{~d}$ postpartum and from nursing piglets through anterior vena cava at $24 \mathrm{~h}$ and $21 \mathrm{~d}$ after birth. Blood samples were centrifuged at 1,700 g at $4^{\circ} \mathrm{C}$ for $15 \mathrm{~min}$ (Eppendorf centrifuge 5810R, Hamburg, Germany) to separate plasma. Colostrum and milk were collected from the first and second teats at $24 \mathrm{~h}$ and $21 \mathrm{~d}$ postpartum after an intravascular injection with 5 IU oxytocin (Komi oxytocin inj. Komipharm International Co., Ltd., Siheung, Korea) in the ear. All samples were stored at $-20^{\circ} \mathrm{C}$ until analysis.

Diets were analyzed for $\mathrm{CP}$ by the Kjedahl method (984.13; AOAC, 2005). Proximate analysis of colostrum and milk was conducted using Milkoscan FT 120 (FOSS Electric, Sungnam, Korea). Plasma urea nitrogen (PUN) level was analyzed using a blood analyzer (Ciba-Corning model, Express Plus, Ciba Corning Diagnostics Co., Massachusetts, US). Plasma free fatty acid (FFA) levels were determined using Wako FFA C Kit (Wako Chemical, Osaka, Japan), and plasma glucose level was detected by an enzymatic kit (Glucose Hexokinase Kit, Bayer, Pennsylvania, US). The amino acid contents in colostrum, milk and diets were determined by ion-exchange chromatography (Amino Acid Analyzer L-8900, Hitachi, Tokyo, Japan) with post-column derivatization with 
Table 1. The formula and chemical composition of experimental diets in gestation and lactation (as-fed basis) ${ }^{1}$

\begin{tabular}{|c|c|c|c|c|c|}
\hline \multirow{2}{*}{ Item } & \multicolumn{4}{|c|}{ Protein level in gestation $(\%)$} & \multirow{2}{*}{ Lactation } \\
\hline & 11.44 & 12.93 & 15.04 & 17.13 & \\
\hline \multicolumn{6}{|l|}{ Ingredients (\%) } \\
\hline Corn & 82.16 & 77.25 & 72.33 & 67.40 & 67.42 \\
\hline Soybean meal (45\% CP) & 8.86 & 13.20 & 17.52 & 21.80 & 24.62 \\
\hline Wheat bran & 3.50 & 3.50 & 3.50 & 3.50 & 2.50 \\
\hline Corn gluten meal & 0.00 & 0.85 & 1.70 & 2.55 & 0.00 \\
\hline Sugar molasses & 0.50 & 0.50 & 0.50 & 0.50 & 1.05 \\
\hline Soybean oil & 0.90 & 1.00 & 1.10 & 1.20 & 1.00 \\
\hline L-lysine $\cdot \mathrm{HCl}$ & 0.40 & 0.27 & 0.13 & 0.00 & 0.38 \\
\hline DL-methionine & 0.07 & 0.02 & 0.00 & 0.00 & 0.00 \\
\hline Dicalcium phosphate & 2.62 & 2.30 & 1.96 & 1.64 & 1.50 \\
\hline Limestone & 0.28 & 0.42 & 0.60 & 0.76 & 0.78 \\
\hline Vitamin premix ${ }^{2}$ & 0.20 & 0.20 & 0.20 & 0.20 & 0.20 \\
\hline Mineral premix ${ }^{3}$ & 0.10 & 0.10 & 0.10 & 0.10 & 0.10 \\
\hline Salt & 0.26 & 0.24 & 0.21 & 0.20 & 0.30 \\
\hline Choline chloride-50 & 0.15 & 0.15 & 0.15 & 0.15 & 0.15 \\
\hline \multicolumn{6}{|c|}{ Analyzed chemical composition (\%) } \\
\hline $\mathrm{CP}$ & 11.44 & 12.93 & 15.04 & 17.13 & \\
\hline \multicolumn{6}{|l|}{ Essential amino acids } \\
\hline $\operatorname{Arg}$ & 0.58 & 0.80 & 0.87 & 1.06 & \\
\hline His & 0.26 & 0.36 & 0.36 & 0.42 & \\
\hline Ile & 0.39 & 0.53 & 0.57 & 0.69 & \\
\hline Leu & 0.97 & 1.31 & 1.42 & 1.69 & \\
\hline Lys & 0.67 & 0.84 & 0.76 & 0.82 & \\
\hline Met & 0.27 & 0.32 & 0.25 & 0.30 & \\
\hline Phe & 0.46 & 0.64 & 0.69 & 0.83 & \\
\hline Thr & 0.38 & 0.54 & 0.55 & 0.66 & \\
\hline Val & 0.55 & 0.74 & 0.77 & 0.91 & \\
\hline \multicolumn{6}{|l|}{ Non-essential amino acids } \\
\hline Ala & 0.58 & 0.78 & 0.82 & 0.96 & \\
\hline Asp & 0.87 & 1.25 & 1.32 & 1.61 & \\
\hline Cys & 0.22 & 0.23 & 0.25 & 0.28 & \\
\hline Glu & 2.01 & 2.75 & 2.84 & 3.42 & \\
\hline Gly & 0.41 & 0.57 & 0.59 & 0.71 & \\
\hline Pro & 0.67 & 0.90 & 0.91 & 1.07 & \\
\hline Ser & 0.48 & 0.69 & 0.70 & 0.83 & \\
\hline Tyr & 0.34 & 0.43 & 0.49 & 0.60 & \\
\hline
\end{tabular}

${ }^{1}$ Daily feed provision in gestation was $2.0 \mathrm{~kg} / \mathrm{d}$ and lactation feed was provided ad libitum.

${ }^{2}$ Provided per kg of diet: vitamin A, $10,000 \mathrm{IU}$; vitamin $\mathrm{D}_{3}, 1,500 \mathrm{IU}$; vitamin E, $35 \mathrm{IU}$; vitamin $\mathrm{K}, 3 \mathrm{mg}$; vitamin $\mathrm{B}_{2}, 4 \mathrm{mg}$; vitamin $\mathrm{B}_{6}, 3 \mathrm{mg}$; vitamin $\mathrm{B}_{12}, 15 \mu \mathrm{g}$; pantothenic acid, $10 \mathrm{mg}$; biotin, $50 \mu \mathrm{g}$; niacin, $20 \mathrm{mg}$; folic acid $500 \mu \mathrm{g}$.

${ }^{3}$ Provided per kg of diet: Fe, $75 \mathrm{mg}$ from $\mathrm{FeSO}_{4} ; \mathrm{Mn}, 20 \mathrm{mg}$ from $\mathrm{MnSO}_{4} ; \mathrm{Zn}, 30 \mathrm{mg}$ from $\mathrm{ZnSO}_{4} ; \mathrm{Cu}, 55 \mathrm{mg}$ from CuSO${ }_{4} ; \mathrm{Se}, 100 \mu \mathrm{g}$ from Na $2 \mathrm{SeO}_{3}$; $\mathrm{Co}, 250 \mathrm{mg}$ from $\mathrm{CoSO}_{4} ; \mathrm{I}, 250 \mu \mathrm{g}$ from $\mathrm{Ca}\left(\mathrm{IO}_{3}\right)_{2}$.

ninhydrin. Performic acid was used in oxidizing amino acids and neutralized with sodium citrate dihydrate, and then hydrolyzed with $6 \mathrm{~N} \mathrm{HCl}$ for $22 \mathrm{~h}$ at $110^{\circ} \mathrm{C}$ to be liberated from the protein. Amino acids were quantified with the internal standard method (amino acid mixture standard solution Type H, Wako Chemical, Osaka, Japan; L-cysteic acid, Tokyo Chemical Industry, Tokyo, Japan; DL-methionine sulfone, Sigma, Missouri, US) by measuring the absorption of reaction products with ninhydrin at $570 \mathrm{~nm}$.

\section{Statistical analysis}

Data were analyzed by ANOVA for a completely randomized design using the GLM procedure of SAS (SAS Institute, 2004). Least squares means were calculated for each independent variable. Orthogonal polynomial contrasts were used to determine linear and quadratic effects by increasing dietary $\mathrm{CP}$ levels in gestation for all 
measurements of sows and piglets. Individual sows and their litters were used as the experimental unit. Blood plasma data were analyzed by repeated measures ANOVA. Alpha level used for the determination of significance for all analysis was 0.05 and tendency for all analysis was $\mathrm{p}>0.05$ and $\mathrm{p}<0.10$.

\section{RESULTS}

One sow was removed from $11 \% \mathrm{CP}$ treatment due to severe delivery problem and her piglets were cross-fostered to balance the number of suckling piglets. Body weight and backfat thickness of gilts in gestation and lactation did not differ among dietary treatments (Table 2). However, BW at $24 \mathrm{~h}$ postpartum $(\mathrm{p}=0.09)$ tended to be linearly increased by increasing dietary $\mathrm{CP}$ level. No differences were observed in ADFI and WEI in lactating sows among dietary treatments.

No significant differences were found in litter size as well as litter and piglet weight at birth and after crossfostering (Table 3). Linear increases on weight of litter and piglet $(\mathrm{p}<0.05)$ at $21 \mathrm{~d}$ of lactation and weight gain of litter $(\mathrm{p}<0.01)$ and piglet $(\mathrm{p}<0.05)$ were observed as dietary CP level increased.

Plasma urea nitrogen level was linearly increased at 15 , $35,70,90(\mathrm{p}<0.01)$ and $110 \mathrm{~d}(\mathrm{p}<0.05)$ of gestation by increasing dietary $\mathrm{CP}$ level in gestation (Table 4), and gestating gilts fed $17 \% \mathrm{CP}$ diet had the highest PUN level at $15,35,70,90$, and $110 \mathrm{~d}$ of gestation among dietary treatments. Plasma FFA levels were increased as dietary CP level increased up to $15 \%$, then decreased with quadratic effects at $15 \mathrm{~d}(\mathrm{p}<0.01)$ and $90 \mathrm{~d}(\mathrm{p}<0.05)$, and a quadratic trend at $70 \mathrm{~d}(\mathrm{p}=0.06)$ of gestation. In plasma glucose level, there were a quadratic effect at $15 \mathrm{~d}(\mathrm{p}<0.05)$ and a similar trend at $70 \mathrm{~d}(\mathrm{p}=0.06)$ of gestation. Plasma values in gestation were changed over time $(\mathrm{p}<0.01)$. Plasma levels of FFA and glucose were the highest at $110 \mathrm{~d}$ of gestation whereas PUN level was the lowest. There was a day $\times$ treatment interaction $(\mathrm{p}<0.05)$ in PUN level in which the difference of PUN levels from $11 \%$ to $17 \% \mathrm{CP}$ treatments decreased at $110 \mathrm{~d}$ of gestation. During lactation (Table 5), a linear increase at $24 \mathrm{~h}$ postpartum $(\mathrm{p}<0.01)$ but a trend of linear decrease at $21 \mathrm{~d}$ of lactation $(\mathrm{p}=0.10)$ were observed in PUN levels by increasing dietary CP level. Quadratic trends in plasma FFA level at $24 \mathrm{~h}$ postpartum ( $\mathrm{p}$ $=0.07)$ and in plasma glucose at $21 \mathrm{~d}$ of lactation $(\mathrm{p}=0.10)$ were observed by increasing dietary CP level. Plasma glucose and PUN levels of lactating sows were changed over time $(\mathrm{p}<0.01)$. The PUN level of sows increased but plasma glucose level decreased from $24 \mathrm{~h}$ postpartum to 21 $\mathrm{d}$ of lactation. There was a day $\times$ treatment interaction $(\mathrm{p}<0.01)$ in PUN level in which a linear increase in PUN levels at $24 \mathrm{~h}$ postpartum by increasing dietary CP levels was not detected at $21 \mathrm{~d}$ of lactation. No differences were detected on PUN and plasma glucose levels of nursing pigs in lactation. Plasma glucose $(\mathrm{p}<0.05)$ and PUN $(\mathrm{p}<0.01)$ levels in nursing pigs were changed over time and PUN level decreased but plasma glucose level increased from birth to $21 \mathrm{~d}$ of lactation.

Dietary CP levels in gestation did not affect colostrum and milk compositions (Table 6). Arginine content in colostrum (Table 7) showed a linear decrease $(p=0.06)$ as

Table 2. Effect of CP levels in gestation diets on BW, backfat thickness and weaning to estrus interval of gilts in gestation and lactation

\begin{tabular}{|c|c|c|c|c|c|c|c|}
\hline \multirow{2}{*}{ Item } & \multicolumn{4}{|c|}{ Protein level in gestation (\%) } & \multirow{2}{*}{ SEM $^{1}$} & \multicolumn{2}{|c|}{ p-value } \\
\hline & 11.44 & 12.93 & 15.04 & 17.13 & & Linear & Quadratic \\
\hline No. of sows & 5 & 7 & 6 & 6 & & & \\
\hline \multicolumn{8}{|l|}{ BW (kg) } \\
\hline At mating & 140.5 & 139.1 & 139.7 & 139.8 & 3.00 & 0.902 & 0.813 \\
\hline $110 \mathrm{~d}$ of gestation & 201.9 & 207.1 & 204.0 & 205.3 & 3.77 & 0.694 & 0.610 \\
\hline Gestation gain (d 0 to 110 ) & 61.4 & 67.9 & 64.3 & 65.5 & 2.08 & 0.377 & 0.213 \\
\hline $24 \mathrm{~h}$ postpartum & 179.6 & 189.3 & 186.7 & 189.7 & 3.40 & 0.095 & 0.338 \\
\hline $21 \mathrm{~d}$ of lactation & 174.1 & 175.2 & 177.6 & 177.8 & 5.67 & 0.611 & 0.940 \\
\hline Lactation change (d 0 to 21 ) & -5.5 & -14.1 & -9.1 & -11.8 & 4.32 & 0.492 & 0.509 \\
\hline \multicolumn{8}{|l|}{ Backfat thickness (mm) } \\
\hline At mating & 20.8 & 21.5 & 19.7 & 20.5 & 1.48 & 0.694 & 0.965 \\
\hline $110 \mathrm{~d}$ of gestation & 25.2 & 27.4 & 24.6 & 27.7 & 1.86 & 0.603 & 0.821 \\
\hline Gestation gain (d 0 to 110 ) & 4.4 & 5.9 & 4.9 & 7.2 & 1.31 & 0.243 & 0.786 \\
\hline $24 \mathrm{~h}$ postpartum & 22.8 & 25.4 & 22.6 & 24.6 & 1.97 & 0.762 & 0.879 \\
\hline $21 \mathrm{~d}$ of lactation & 19.2 & 20.9 & 19.9 & 22.2 & 1.72 & 0.333 & 0.881 \\
\hline Lactation change (d 0 to 21 ) & -3.6 & -4.4 & -2.7 & -2.4 & 0.92 & 0.224 & 0.563 \\
\hline Lactation feed intake $(\mathrm{kg} / \mathrm{d})$ & 3.75 & 4.11 & 4.17 & 4.08 & 0.457 & 0.626 & 0.626 \\
\hline Weaning to estrus interval (d) & 5.2 & 5.1 & 5.3 & 5.0 & 0.42 & 0.836 & 0.746 \\
\hline
\end{tabular}

${ }^{1}$ Standard error of the mean. 
Table 3. Effect of CP levels in gestation diets on litter size and litter performance of primiparous sows

\begin{tabular}{|c|c|c|c|c|c|c|c|}
\hline \multirow{2}{*}{ Item } & \multicolumn{4}{|c|}{ Protein level in gestation (\%) } & \multirow{2}{*}{ SEM $^{1}$} & \multicolumn{2}{|c|}{ p-value } \\
\hline & 11.44 & 12.93 & 15.04 & 17.13 & & Linear & Quadratic \\
\hline No. of sows & 5 & 7 & 6 & 6 & & & \\
\hline \multicolumn{8}{|l|}{ Litter size, no. of piglets } \\
\hline Total born & 12.20 & 11.86 & 12.00 & 10.50 & 0.806 & 0.199 & 0.482 \\
\hline Stillbirth & 0.40 & 0.29 & 0.67 & 0.00 & 0.259 & 0.502 & 0.300 \\
\hline Mummified & 0.00 & 0.00 & 0.00 & 0.00 & - & - & - \\
\hline Born alive & 11.80 & 11.57 & 11.33 & 10.50 & 0.838 & 0.299 & 0.722 \\
\hline After cross-fostering & 11.60 & 11.43 & 11.50 & 11.50 & 0.223 & 0.827 & 0.706 \\
\hline Weaning pigs (d 21) & 11.00 & 11.14 & 11.33 & 11.17 & 0.243 & 0.547 & 0.533 \\
\hline Preweaning mortality (\%) & 5.00 & 2.38 & 1.52 & 2.90 & 1.723 & 0.381 & 0.260 \\
\hline \multicolumn{8}{|l|}{ Litter weight $(\mathrm{kg})$} \\
\hline At birth & 16.20 & 15.46 & 16.13 & 13.63 & 1.121 & 0.193 & 0.445 \\
\hline After cross-fostering & 14.58 & 14.91 & 15.52 & 15.22 & 0.764 & 0.486 & 0.683 \\
\hline $21 \mathrm{~d}$ of lactation & 46.72 & 56.40 & 51.87 & 58.98 & 2.498 & 0.011 & 0.614 \\
\hline Litter weight gain (d 0 to $21, \mathrm{~kg} / \mathrm{d}$ ) & 1.54 & 1.96 & 1.72 & 2.10 & 0.108 & 0.008 & 0.874 \\
\hline \multicolumn{8}{|l|}{ Piglet weight $(\mathrm{kg})$} \\
\hline At birth & 1.29 & 1.33 & 1.35 & 1.33 & 0.067 & 0.651 & 0.656 \\
\hline After cross-fostering & 1.27 & 1.33 & 1.35 & 1.33 & 0.067 & 0.482 & 0.593 \\
\hline $21 \mathrm{~d}$ of lactation & 4.25 & 5.06 & 4.60 & 5.31 & 0.257 & 0.032 & 0.844 \\
\hline Piglet weight gain (d 0 to $21, \mathrm{~kg} / \mathrm{d}$ ) & 0.14 & 0.18 & 0.16 & 0.19 & 0.011 & 0.035 & 0.952 \\
\hline
\end{tabular}

${ }^{1}$ Standard error of mean.

dietary CP level increased. Histidine $(\mathrm{p}<0.05)$ content increased with a quadratic effect. Isoleucine $(\mathrm{p}<0.05)$ and decreased as dietary CP level increased up to $15 \%$, then lysine content tended to decrease as dietary $\mathrm{CP}$ level

Table 4. Effect of CP levels in gestation diets on blood profiles of gilts in gestation ${ }^{1}$

\begin{tabular}{|c|c|c|c|c|c|c|c|}
\hline \multirow{2}{*}{ Item } & \multicolumn{4}{|c|}{ Protein level in gestation (\%) } & \multirow{2}{*}{ SEM $^{2}$} & \multicolumn{2}{|c|}{ p-value } \\
\hline & 11.44 & 12.93 & 15.04 & 17.13 & & Linear & Quadratic \\
\hline \multicolumn{8}{|c|}{ Plasma urea nitrogen $(\mathrm{g} / \mathrm{dL})^{3,4}$} \\
\hline At mating ${ }^{5}$ & 10.9 & 10.9 & 10.9 & 10.9 & - & - & - \\
\hline $15 \mathrm{~d}$ & 9.2 & 10.3 & 12.2 & 15.1 & 0.71 & $<0.001$ & 0.225 \\
\hline $35 \mathrm{~d}$ & 8.6 & 10.2 & 12.0 & 16.0 & 0.76 & $<0.001$ & 0.130 \\
\hline $70 \mathrm{~d}$ & 7.0 & 9.8 & 11.8 & 15.6 & 0.68 & $<0.001$ & 0.447 \\
\hline $90 \mathrm{~d}$ & 6.2 & 7.7 & 10.1 & 13.1 & 0.42 & $<0.001$ & 0.113 \\
\hline $110 \mathrm{~d}$ & 8.2 & 7.8 & 10.0 & 11.0 & 1.02 & 0.031 & 0.510 \\
\hline \multicolumn{8}{|c|}{ Plasma free fatty acids $(\mu \mathrm{Eq} / \mathrm{L})^{3}$} \\
\hline At mating & 69.6 & 69.6 & 69.6 & 69.6 & - & - & - \\
\hline $15 \mathrm{~d}$ & 33.4 & 42.4 & 45.8 & 29.0 & 4.10 & 0.600 & 0.006 \\
\hline $35 \mathrm{~d}$ & 29.6 & 31.8 & 33.2 & 26.0 & 3.16 & 0.515 & 0.156 \\
\hline $70 \mathrm{~d}$ & 29.4 & 34.4 & 36.8 & 27.4 & 3.56 & 0.824 & 0.061 \\
\hline $90 \mathrm{~d}$ & 32.2 & 26.8 & 68.2 & 34.2 & 6.26 & 0.110 & 0.037 \\
\hline $110 \mathrm{~d}$ & 108.0 & 127.4 & 132.6 & 85.5 & 6.06 & 0.356 & 0.794 \\
\hline \multicolumn{8}{|c|}{ Plasma glucose $(\mathrm{mg} / \mathrm{dL})^{3}$} \\
\hline At mating & 73.0 & 73.0 & 73.0 & 73.0 & - & - & - \\
\hline $15 \mathrm{~d}$ & 61.0 & 68.4 & 68.2 & 63.2 & 2.92 & 0.631 & 0.050 \\
\hline $35 \mathrm{~d}$ & 63.4 & 70.6 & 67.6 & 58.4 & 4.84 & 0.418 & 0.110 \\
\hline $70 \mathrm{~d}$ & 70.2 & 64.4 & 54.2 & 65.6 & 4.19 & 0.218 & 0.057 \\
\hline $90 \mathrm{~d}$ & 67.0 & 61.6 & 69.4 & 64.6 & 3.52 & 0.970 & 0.933 \\
\hline $110 \mathrm{~d}$ & 78.4 & 77.6 & 73.2 & 76.4 & 6.06 & 0.706 & 0.746 \\
\hline
\end{tabular}

${ }^{1}$ Least squares means of 5 observations per treatment. ${ }^{2}$ Standard error of mean.

${ }^{3}$ Day effect ( $\mathrm{p}<0.01$; repeated measures ANOVA). ${ }^{4}$ Day $\times$ treatment interaction $(\mathrm{p}<0.05$; repeated measures ANOVA).

${ }^{5}$ Blood samples $(\mathrm{n}=11)$ were randomly taken from gilts immediately before mating. 
Table 5. Effect of CP levels in gestation diets on blood profiles of primiparous sows and their progeny in lactation ${ }^{1}$

\begin{tabular}{|c|c|c|c|c|c|c|c|}
\hline \multirow{2}{*}{ Item } & \multicolumn{4}{|c|}{ Protein level in gestation (\%) } & \multirow{2}{*}{ SEM $^{2}$} & \multicolumn{2}{|c|}{ p-value } \\
\hline & 11.44 & 12.93 & 15.04 & 17.13 & & Linear & Quadratic \\
\hline \multicolumn{8}{|l|}{ Sows } \\
\hline \multicolumn{8}{|c|}{ Plasma urea nitrogen $(\mathrm{g} / \mathrm{dL})^{3,5}$} \\
\hline $24 \mathrm{~h}$ postpartum & 8.8 & 11.7 & 13.8 & 14.6 & 0.96 & 0.001 & 0.294 \\
\hline $21 \mathrm{~d}$ of lactation & 15.8 & 15.8 & 15.9 & 14.4 & 0.97 & 0.095 & 0.783 \\
\hline \multicolumn{8}{|c|}{ Plasma free fatty acids $(\mu \mathrm{Eq} / \mathrm{L})$} \\
\hline $24 \mathrm{~h}$ postpartum & 333.5 & 187.6 & 127.0 & 229.6 & 60.21 & 0.254 & 0.068 \\
\hline $21 \mathrm{~d}$ of lactation & 164.6 & 335.8 & 200.8 & 163.5 & 68.30 & 0.625 & 0.194 \\
\hline \multicolumn{8}{|c|}{ Plasma glucose $(\mathrm{mg} / \mathrm{dL})^{3}$} \\
\hline $24 \mathrm{~h}$ postpartum & 97.8 & 89.0 & 99.0 & 92.2 & 6.04 & 0.804 & 0.871 \\
\hline $21 \mathrm{~d}$ of lactation & 83.6 & 87.6 & 85.0 & 79.2 & 2.78 & 0.222 & 0.097 \\
\hline \multicolumn{8}{|l|}{ Nursing pigs } \\
\hline \multicolumn{8}{|c|}{ Plasma urea nitrogen $(\mathrm{g} / \mathrm{dL})^{3}$} \\
\hline At birth & 16.4 & 19.1 & 14.5 & 19.1 & 2.32 & 0.724 & 0.684 \\
\hline $21 \mathrm{~d}$ of lactation & 10.2 & 7.9 & 7.6 & 9.7 & 1.65 & 0.833 & 0.206 \\
\hline \multicolumn{8}{|c|}{ Plasma glucose $(\mathrm{mg} / \mathrm{dL})^{4}$} \\
\hline At birth & 97.6 & 98.0 & 92.0 & 96.0 & 13.13 & 0.856 & 0.893 \\
\hline $21 \mathrm{~d}$ of lactation & 116.8 & 118.6 & 105.8 & 115.0 & 4.77 & 0.407 & 0.450 \\
\hline
\end{tabular}

${ }^{1}$ Least squares means of 5 observations per treatment. ${ }^{2}$ Standard error of mean.

${ }^{3}$ Day effect ( $\mathrm{p}<0.01$; repeated measures ANOVA). ${ }^{4}$ Day effect $(\mathrm{p}<0.05$; repeated measures ANOVA).

${ }^{5}$ Day $\times$ treatment interaction ( $<<0.01$; repeated measures ANOVA).

increased up to $13 \%(\mathrm{p}=0.09)$, then increased with quadratic effects. With a quadratic effect $(\mathrm{p}<0.05)$ the highest cysteine concentration was observed when gilts were fed the $15 \% \mathrm{CP}$ diet in gestation than the other treatment diets. Glycine $(\mathrm{p}<0.05)$ content increased as dietary CP level increased up to $13 \%$. Quadratic increases were observed with trends in serine $(\mathrm{p}=0.06)$ and tyrosine $(\mathrm{p}=0.08)$ contents as dietary $\mathrm{CP}$ level increased up to $15 \%$. In milk (Table 8), linear decreases were observed in arginine $(\mathrm{p}<0.01)$, methionine $(\mathrm{p}<0.01)$ and cysteine $(p<0.05)$ content by increasing dietary CP level. Glutamic acid $(\mathrm{p}<0.05)$ and proline $(\mathrm{p}=0.07)$ content increased linearly by increasing CP levels.

\section{DISCUSSION}

Effect of high protein intake in gestation has been reported to increase milk production and $\mathrm{BW}$ at farrowing, and improve subsequent reproductive performance of gilts (Belstra et al., 1998; Mahan, 1998; Kusina et al., 1999b). In the current study, BW of gilts at farrowing tended to be linearly increased by increasing CP level. Previous studies consistently reported high protein intake in gestation increased sow BW at farrowing, and weight gain in

Table 6. Effect of CP levels in gestation diets on milk composition of primiparous sows ${ }^{1}$

\begin{tabular}{|c|c|c|c|c|c|c|c|}
\hline \multirow{2}{*}{ Item } & \multicolumn{4}{|c|}{ Protein level in gestation $(\%)$} & \multirow{2}{*}{$\mathrm{SEM}^{2}$} & \multicolumn{2}{|c|}{$\mathrm{p}$-value } \\
\hline & 11.44 & 12.93 & 15.04 & 17.13 & & Linear & Quadratic \\
\hline \multicolumn{8}{|c|}{ Colostrum at $24 \mathrm{~h}$ postpartum (\%) } \\
\hline Fat & 8.78 & 6.99 & 7.15 & 7.32 & 0.869 & 0.104 & 0.138 \\
\hline Protein & 8.01 & 8.64 & 7.79 & 7.41 & 1.145 & 0.611 & 0.664 \\
\hline Lactose & 3.80 & 3.89 & 4.03 & 4.04 & 0.227 & 0.415 & 0.862 \\
\hline Total solids & 25.68 & 22.86 & 22.05 & 21.84 & 1.896 & 0.165 & 0.523 \\
\hline Solids not fat & 11.58 & 12.30 & 11.75 & 11.36 & 0.813 & 0.743 & 0.501 \\
\hline \multicolumn{8}{|c|}{ Milk at $21 \mathrm{~d}$ of lactation (\%) } \\
\hline Fat & 6.77 & 6.97 & 6.76 & 6.69 & 0.400 & 0.808 & 0.744 \\
\hline Protein & 4.85 & 4.64 & 4.78 & 4.83 & 0.121 & 0.871 & 0.304 \\
\hline Lactose & 5.40 & 5.59 & 5.52 & 5.58 & 0.065 & 0.117 & 0.301 \\
\hline Total solids & 19.13 & 19.22 & 19.05 & 19.19 & 0.450 & 0.995 & 0.955 \\
\hline Solids not fat & 10.31 & 10.21 & 10.27 & 10.39 & 0.120 & 0.578 & 0.387 \\
\hline
\end{tabular}

${ }^{1}$ Least squares means of 5 observations per treatment. ${ }^{2}$ Standard error of mean. 
Table 7. Effect of CP levels in gestation diets on amino acid composition ( $\mathrm{g} / 100 \mathrm{~g}$ milk protein) in colostrum of primiparous sows ${ }^{1}$

\begin{tabular}{|c|c|c|c|c|c|c|c|}
\hline \multirow{2}{*}{ Item } & \multicolumn{4}{|c|}{ Protein level in gestation $(\%)$} & \multirow{2}{*}{ SEM $^{2}$} & \multicolumn{2}{|c|}{ p-value } \\
\hline & 11.44 & 12.93 & 15.04 & 17.13 & & Linear & Quadratic \\
\hline \multicolumn{8}{|c|}{ Essential amino acids } \\
\hline Arg & 4.94 & 4.91 & 4.85 & 4.70 & 0.080 & 0.057 & 0.461 \\
\hline His & 2.72 & 2.63 & 2.60 & 2.73 & 0.040 & 0.958 & 0.021 \\
\hline Ile & 3.68 & 3.49 & 3.51 & 3.70 & 0.071 & 0.778 & 0.023 \\
\hline Leu & 9.13 & 9.27 & 9.10 & 9.26 & 0.135 & 0.710 & 0.954 \\
\hline Lys & 7.24 & 7.09 & 7.12 & 7.37 & 0.108 & 0.428 & 0.093 \\
\hline Met & 2.15 & 1.90 & 2.03 & 1.95 & 0.078 & 0.239 & 0.284 \\
\hline Phe & 4.20 & 4.24 & 4.25 & 4.18 & 0.080 & 0.932 & 0.493 \\
\hline Thr & 5.20 & 5.41 & 5.48 & 5.19 & 0.172 & 0.941 & 0.177 \\
\hline Val & 6.72 & 6.77 & 6.67 & 6.69 & 0.193 & 0.846 & 0.945 \\
\hline \multicolumn{8}{|c|}{ Non-essential amino acids } \\
\hline Ala & 4.08 & 4.14 & 4.14 & 4.14 & 0.050 & 0.362 & 0.552 \\
\hline Asp & 8.27 & 8.09 & 8.12 & 8.15 & 0.121 & 0.582 & 0.412 \\
\hline Cys & 1.74 & 1.77 & 1.83 & 1.53 & 0.054 & 0.040 & 0.011 \\
\hline Glu & 17.67 & 17.41 & 17.38 & 18.19 & 0.482 & 0.503 & 0.289 \\
\hline Gly & 3.11 & 3.29 & 3.25 & 3.05 & 0.083 & 0.586 & 0.047 \\
\hline Pro & 8.93 & 9.10 & 8.89 & 9.13 & 0.143 & 0.569 & 0.840 \\
\hline Ser & 5.78 & 5.97 & 6.05 & 5.70 & 0.125 & 0.811 & 0.059 \\
\hline Tyr & 4.49 & 4.54 & 4.74 & 4.33 & 0.121 & 0.657 & 0.080 \\
\hline
\end{tabular}

${ }^{1}$ Least squares means of 5 observations per treatment. ${ }^{2}$ Standard error of mean.

gestation (Mahan and Mangan, 1975; Heo et al., 2008; Zhang et al., 2011), indicating that increasing protein intake in gestation could increase body reserves with protein deposition (Mahan, 1998; Kusina et al., 1999b). Similarly, Jones and Maxwell (1982) reported a quadratic increase of daily weight gain of gilts by increasing protein intake in gestation even though protein intake did not affect BW of gilts. Therefore, the increase of protein intake in gestation could increase body protein reserves of gestating gilts through the slight increase of BW.

Table 8. Effect of $\mathrm{CP}$ levels in gestation diets on amino acid composition ( $\mathrm{g} / 100 \mathrm{~g}$ milk protein) in milk of primiparous sows at $21 \mathrm{~d}$ of lactation $^{1}$

\begin{tabular}{|c|c|c|c|c|c|c|c|}
\hline \multirow{2}{*}{ Item } & \multicolumn{4}{|c|}{ Protein level in gestation (\%) } & \multirow{2}{*}{$\mathrm{SEM}^{2}$} & \multicolumn{2}{|c|}{$\mathrm{p}$-value } \\
\hline & 11.44 & 12.93 & 15.04 & 17.13 & & Linear & Quadratic \\
\hline \multicolumn{8}{|c|}{ Essential amino acids } \\
\hline Arg & 4.59 & 4.64 & 4.52 & 4.43 & 0.040 & 0.009 & 0.145 \\
\hline His & 2.67 & 2.72 & 2.69 & 2.67 & 0.038 & 0.944 & 0.379 \\
\hline Ile & 3.93 & 3.93 & 3.94 & 3.84 & 0.051 & 0.307 & 0.377 \\
\hline Leu & 8.56 & 8.36 & 8.51 & 8.61 & 0.097 & 0.492 & 0.153 \\
\hline Lys & 7.58 & 7.55 & 7.59 & 7.50 & 0.059 & 0.490 & 0.642 \\
\hline Met & 2.54 & 2.02 & 2.22 & 1.84 & 0.101 & 0.002 & 0.505 \\
\hline Phe & 3.84 & 3.78 & 3.80 & 3.84 & 0.038 & 0.847 & 0.188 \\
\hline Thr & 4.26 & 4.13 & 4.23 & 4.23 & 0.040 & 0.751 & 0.119 \\
\hline Val & 5.89 & 5.99 & 5.96 & 5.94 & 0.048 & 0.538 & 0.229 \\
\hline \multicolumn{8}{|c|}{ Non-essential amino acids } \\
\hline Ala & 3.51 & 3.53 & 3.56 & 3.52 & 0.055 & 0.811 & 0.697 \\
\hline Asp & 8.11 & 8.06 & 8.03 & 7.96 & 0.067 & 0.151 & 0.836 \\
\hline Cys & 1.56 & 1.46 & 1.50 & 1.24 & 0.071 & 0.019 & 0.297 \\
\hline Glu & 20.42 & 20.91 & 20.72 & 21.13 & 0.139 & 0.012 & 0.751 \\
\hline Gly & 2.96 & 3.08 & 2.98 & 3.07 & 0.067 & 0.491 & 0.837 \\
\hline Pro & 10.47 & 10.84 & 10.58 & 11.09 & 0.174 & 0.071 & 0.690 \\
\hline Ser & 5.15 & 5.04 & 5.19 & 5.15 & 0.041 & 0.419 & 0.393 \\
\hline Tyr & 3.98 & 3.98 & 3.99 & 3.90 & 0.081 & 0.575 & 0.637 \\
\hline
\end{tabular}

${ }^{1}$ Least squares means of 5 observations per treatment. ${ }^{2}$ Standard error of mean. 
When gestating gilts were fed $11 \% \mathrm{CP}$ diet, the lowest BW change was observed numerically in overall lactation among dietary treatments. Heo et al. (2008) reported a high protein intake in gestation and lactation reduced sow BW loss in lactation. However, in this study, the sows consumed the same lactation diet during lactation. Thus, this observation might be associated with ADFI of sows and litter growth in lactation. Even though ADFI of sows in lactation did not differ by dietary CP levels, the lowest litter performance was observed when gilts were fed $11 \% \mathrm{CP}$ diet in gestation. Therefore, it is likely that low piglet growth in $11 \% \mathrm{CP}$ treatment with similar feed intake may result in lower maternal BW loss compared with the other treatments.

Litter size and WEI were not affected by dietary treatments in the current study, which agreed with previous studies. Tydlitat et al. (2008) reported increasing protein level in gestation diets did not affect total number born but resulted in decreased total number of born alive and increased stillborn piglet. Shields et al. (1985) also reported similar results in which litter size was not affected by dietary protein level in gestation. Concerning WEI, Greenhalgh et al. (1977) observed a longer WEI in sows fed low protein diet for gestation (9\% CP) and lactation (13\% $\mathrm{CP})$ compared with those fed high protein diet for gestation $(11 \%$ or $13 \% \mathrm{CP})$ and lactation $(17 \% \mathrm{CP})$. The WEI could be delayed due to low plasma insulin concentration in lactation, and high glucose but low FFA concentrations in plasma of weaning sows (Armstrong et al., 1986) as well as high maternal protein loss during lactation (Clowes et al., 2003), resulting in a reduced subsequent reproductive performance. In this study, plasma glucose and FFA concentrations of sows at weaning had no considerable differences among dietary treatments, indicating that dietary protein level did not affect WEI.

In the current study, piglet birth weight was not affected by dietary CP level, which agreed with Tydlitat et al. (2008) who reported mean birth weight of piglets did not differ by dietary protein levels in gestation. Even though low CP level $(11 \%)$ in gestation diet showed no negative effects on litter and piglet birth weight in this study, litter growth was the lowest among dietary treatments, which agreed with the previous work of Mahan (1998) who reported high dietary $\mathrm{CP}$ levels with high feed intake in gestation increased litter weight and gain, more in primiparous sows than old sows. Based on the results of the current study, $11 \% \mathrm{CP}$ level in the gestation diet under equal lysine content was not enough to maximize litter growth which may be attributed to lack of the other essential amino acids available in the diet compared with the other diets. Insufficient amino acid content in gestation diets caused failure to normal litter growth in lactation, because of inadequate body protein reserves to produce milk, limitation of mammary gland development and damage to fetuses during gestation (Kusina et al., 1999a). Therefore, high CP level had a positive influence on litter growth but $11 \% \mathrm{CP}$ level in gestation diet was not sufficient for gestating gilts to maximize piglet growth.

The PUN level in gestation increased linearly by increasing CP level, which agreed with Kusina et al. (1999b) who reported that PUN level increased by increasing protein content in gestation diets. The PUN is a good indicator of protein utilization in animal body and low concentration of blood urea may be derived from reduced availability of ammonia caused by enhanced protein synthesis and reduced amino acid oxidation (Wu and Morris, 1998). In this study, with equal lysine content $11 \%$ CP diet showed the lowest PUN level in gestation, meaning that protein utilization was the highest among dietary treatments. Additionally, the reduced patterns of PUN levels as gestation progressed indicated protein utilization increased in late gestation. The PUN is affected by several factors such as genders, feeding methods, protein level and quality (Eggum, 1970). A partial replacement of protein sources by animal- or plant-based protein ingredients had no effect on PUN concentration in pigs (Sun et al., 2009) whereas protein level in the diet had a positive correlation with PUN concentration in pigs (Eggum, 1970). In this study, dietary protein level was adjusted by cereal grains (soybean meal and corn gluten meal), and gilts fed high protein diets in gestation showed high PUN level throughout gestation with linear increases by dietary protein levels, which agreed with these previous studies. On the other hand, plasma values of sows at $21 \mathrm{~d}$ of lactation were not significantly different among dietary treatments because lactating sows were fed the same diet ad libitum and lactation feed intake was not different among dietary treatments. In addition, PUN and plasma glucose level of suckling pigs were not affected by protein levels in gestation diets, which resulted from similar milk composition among treatments. These results indicated that there were no major effects on blood profiles of lactating sows and their progeny by increasing protein level in gestation diets, which was in agreement with Heo et al. (2008).

In this study, plasma FFA levels were the highest at 110 $\mathrm{d}$ of gestation and the gestating gilts fed 15\% CP diet showed the highest plasma FFA level in gestation with quadratic responses. The FFA levels were represented for satisfying energy demands and reflected fat mobilization from body reserves (Chilliard, 1993). Belstra et al. (1998) reported that plasma FFA level in gestation reached a peak level when energy needs for sows were the greatest, and that it reflected body fat mobilization. These results might indicate the energetic requirement of sows during gestation was the highest in late gestation and increased as dietary $\mathrm{CP}$ 
level increased up to $15 \%$ for mammary gland development, and thereby those sows could have high litter growth in lactation. However, there are a few evidences about the effect of dietary protein level on blood FFA of sows, thus further study is needed to demonstrate this effect clearly.

In the current study, dietary $\mathrm{CP}$ levels in gestation diets did not affect proximate composition of colostrum and milk. In contrast, it has been reported that high protein intake in gestation increased protein content in colostrum (Heo et al., 2008; Zhang et al., 2011). In this study, quadratic effects on histidine, isoleucine, cysteine, glycine, and with tendencies lysine, serine and tyrosine content in colostrum and linear effects on arginine, methionine, cysteine, glutamic acid, and with a tendency proline in milk were observed as dietary $\mathrm{CP}$ level increased in gestation but the results were variable by dietary CP level. Elliott et al. (1971) reported that milk composition was not affected by dietary factors, especially protein but essential amino acids in colostrum of sows fed low $\mathrm{CP}$ diet were the lowest and increased by increasing dietary protein level. Consequently, the litter growth was linearly increased by increasing dietary CP levels. Based on these results, increased litter growth by increasing CP levels in gestation diets might be explained by increase of milk yield, not improvement of milk composition. Further study is needed to clearly demonstrate a possible correlation between protein level in gestation and milk production and quality.

\section{IMPLICATIONS}

Several effects of increasing protein levels in gestation diets were observed in reproductive performance and blood parameters even though lysine content was similar between dietary treatments: i) High CP content in gestation diets for gilts had positive effects on sow body reserves. ii) Increased dietary CP level in gestation for gilts improved litter growth during lactation but did not affect litter size and litter birth weight. iii) The PUN level in gestation increased as dietary CP level increased but plasma FFA levels were the highest when $15 \% \mathrm{CP}$ diet was provided to gestating gilts. Consequently, above $13 \% \mathrm{CP}$ may be recommended for gestating gilts to maximize piglet growth.

\section{ACKNOWLEDGEMENT}

This study was supported by Rural Development Administration (RDA; Project No. PJ009226), Republic of Korea.

\section{REFERENCES}

AOAC. 2005. Official methods of analysis, 18th edition.
Association of Official Analytical Chemists, Washington DC, US.

Armstrong, J. D., J. H. Britt, and R. R. Kraeling. 1986. Effect of restriction of energy during lactation on body condition, energy metabolism, endocrine changes and reproductive performance in primiparous sows. J. Anim. Sci. 63:1915-1925.

Baker, D. H., D. E. Becker, A. H. Jensen, and B. G. Harman. 1970. Reproductive performance and progeny development in swine as influenced by protein restriction during various portions of gestation. J. Anim. Sci. 31:526-530.

Belstra, B. A., B. T. Richert, and J. W. Frank. 1998. Effect of gestation dietary crude protein level on the gestation and lactation performance of primiparous sows. Purdue University 1998 Swine Day Reports. pp. 60-64.

Boyd, R. D., K. J. Touchette, G. C. Castro, M. E. Johnston, K. U. Lee, and In K. Han. 2000. Recent advances in amino acid and energy nutrition of prolific sows. Asian-Aus. J. Anim. Sci. 13: 1638-1652.

Chilliard, Y. 1993. Dietary fat and adipose tissue metabolism in ruminants, pigs, and rodents: A review. J. Dairy Sci. 76:38973931.

Clowes, E. J., F. X. Aherne, G. R. Foxcroft, and V. E. Barocos. 2003. Selective protein loss in lactating sows is associated with reduced litter growth and ovarian function. J. Anim. Sci. 81: 753-764.

Eggum, B. O. 1970. Blood urea measurement as a technique for assessing protein quality. Br. J. Nutr. 24:983-988.

Elliott, R. F., G. W. Vander Noot, R. L. Gilbreath, and H. Fisher. 1971. Effect of dietary protein level on composition changes in sow colostrum and milk. J. Anim. Sci. 32: 1128-1137.

Greenhalgh, J. F. D., F. W. H. Elsley, D. A. Grubb, A. L. Lightfoot, D. W. Saul, P. Smith, N. Walker, D. Williams, and M. L. Yeo. 1977. Coordinated trials on the protein requirements of sows. 1. A comparison of four levels of dietary protein in gestation and two in lactation. Anim. Prod. 24:307-321.

Heo, S., Y. X. Yang, Z. Jin, M. S. Park, B. K. Yang, and B. J. Chae. 2008. Effects of dietary energy and lysine intake during late gestation and lactation on blood metabolites, hormones, milk composition and reproductive performance in primiparous sows. Can. J. Anim. Sci. 88:247-255.

Jones, R. D. and C. V. Maxwell. 1982. Growth, reproductive performance and nitrogen balance of gilts as affected by protein intake and stage of gestation. J. Anim. Sci. 55:848-856.

Kim, S. W., W. L. Hurley, G. Wu, and F. Ji. 2009. Ideal amino acid balance for sows during gestation and lactation. J. Anim. Sci. 87:E123-E132.

Kusina, J., J. E. Pettigrew, A. F. Sower, M. R. Hathaway, M. E. White, and B. A. Crooker. 1999a. Effect of protein intake during gestation on mammary development of primiparous sows. J. Anim. Sci. 77:925-930.

Kusina, J., J. E. Pettigrew, A. F. Sower, M. E. White, B. A. Crooker, and M. R. Hathaway. 1999b. Effect of protein intake during gestation and lactation on the lactational performance of primiparous sows. J. Anim. Sci. 77:931-941.

Mahan, D. C. and L. T. Mangan. 1975. Evaluation of various protein sequences on the nutritional carry-over from gestation to lactation with first-litter sows. J. Nutr. 105:1291-1298.

Mahan, D. C. 1998. Relationship of gestation protein and feed 
intake level over a five-parity period using a high-producing sow genotype. J. Anim. Sci. 76:533-541.

National Research Council. 1998. Nutrient requirements of swine, 10th Ed. National Academy Press, Washington, DC.

Pettigrew, J. E. and H. Yang. 1997. Protein nutrition of gestating sows. J. Anim. Sci. 75:2723-2730.

SAS Institute Inc. 2004. SAS/STAT user's guide, SAS Institute Inc., Cary. NC.

Shields, R. G., Jr., D. C. Mahan, and P. F. Maxson. 1985. Effect of dietary gestation and lactation protein levels on reproductive performance and body composition of first-litter female swine. J. Anim. Sci. 60:179-189.

Sun, Z., Q. Ma, Z. Li, and C. Ji. 2009. Effect of partial substitution of dietary spray-dried porcine plasma or fishmeal with soybean and shrimp protein hydrolysate on growth performance, nutrient digestibility and serum biochemical parameters of weaning piglets. Asian-Aust. J. Anim. Sci. 22:1032-1037.
Tydlitat, D., A. Vinkler, and L. Czanderlova. 2008. Influence of crude protein intake on the duration of delivery and litter size in sows. Acta Vet. Brno. 77:25-30.

Valros, A., M. Rundgren, M. Špinka, H. Saloniemi, L. Rydhmer, F. Hultén, K. Uvnäs-Moberg, M. Tománek, P. Krejcí, and B. Algers. 2003. Metabolic state of the sow, nursing behaviour and milk production. Livest. Prod. Sci. 79:155-167.

Wu, G. and S. M. Morris Jr. 1998. Arginine metabolism: Nitric oxide and beyond. Biochem. J. 336:1-17.

Zhang, R. F., Q. Hu, P. F. Li, L. F. Xue, X. S. Piao, and D. F. Li. 2011. Effects of lysine intake during middle to late gestation (day 30 to 110) on reproductive performance, colostrum composition, blood metabolites and hormones of multiparous sows. Asian-Aust. J. Anim. Sci. 24:1142-1147. 